\title{
Rapidly progressive ulcer in an older woman
}

A fit and well 78-year-old woman with no significant past medical history presented to the Emergency Department with large painless perianal ulcers. It started as an erythematous patch that developed into pruritic perianal blisters, and rapidly evolved into a necrotic ulcer over 2 days. She denied any prior injury or new contacts and was not on any new medications. At presentation, she was afebrile and physical examination findings were unremarkable except for the large $16 \times 15 \mathrm{~cm}$ gangrenous ulcer with a black scab surrounded by an erythematous halo on the perianal region extending to the intergluteal cleft. There was another similar ulcer on the left gluteal measuring $5 \times 3 \mathrm{~cm}$ (Fig. 1A). Biochemical investigations showed normal leukocyte count of $9.71 \times 10^{9} / \mathrm{L}$ (reference range, $3.37-10.93 \times 10^{9} / \mathrm{L}$ ) and a $\mathrm{C}$-reactive protein level of $7.1 \mathrm{mg} / \mathrm{L}$ (reference range $<5.0 \mathrm{mg} / \mathrm{L}$ ). The patient had no bacteraemia. Punch biopsy was done for histological and microbiological examination.
What is the diagnosis?
A. Necrotising fasciitis of the perineum
B. Aspergillosis-related primary cutaneous ulceration
C. Rapidly developing sacral sore
D. Ecthyma gangrenosum in immunocompetent individual
E. Mucormycosis

Histology showed fibro-adipose tissue showing almost complete necrosis, mostly bland. Multiple zones of suppuration, accompanied by fungal organisms with branching hyphae (Fig. 2). No granulomatous inflammation was seen. No malignancy was identified. Initial tissue culture grew Aspergillus flavus and Candida tropicalis. She was diagnosed with ecthyma gangrenosum.

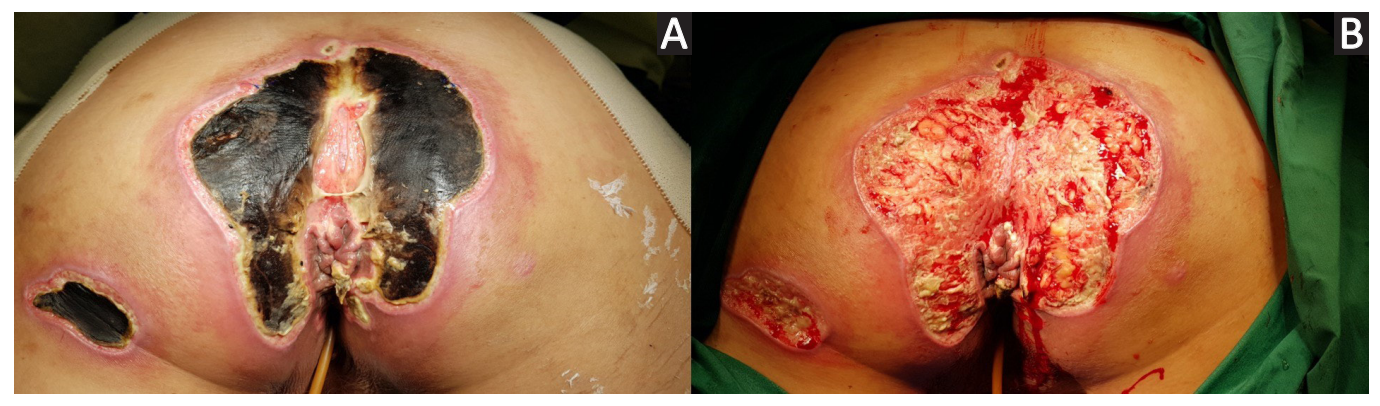

Fig. 1. Perianal necrotic ulcers prior to debridement. (A) Post debridement showing healthy adipose tissue with good vascularity, signifying a superficial process (B).

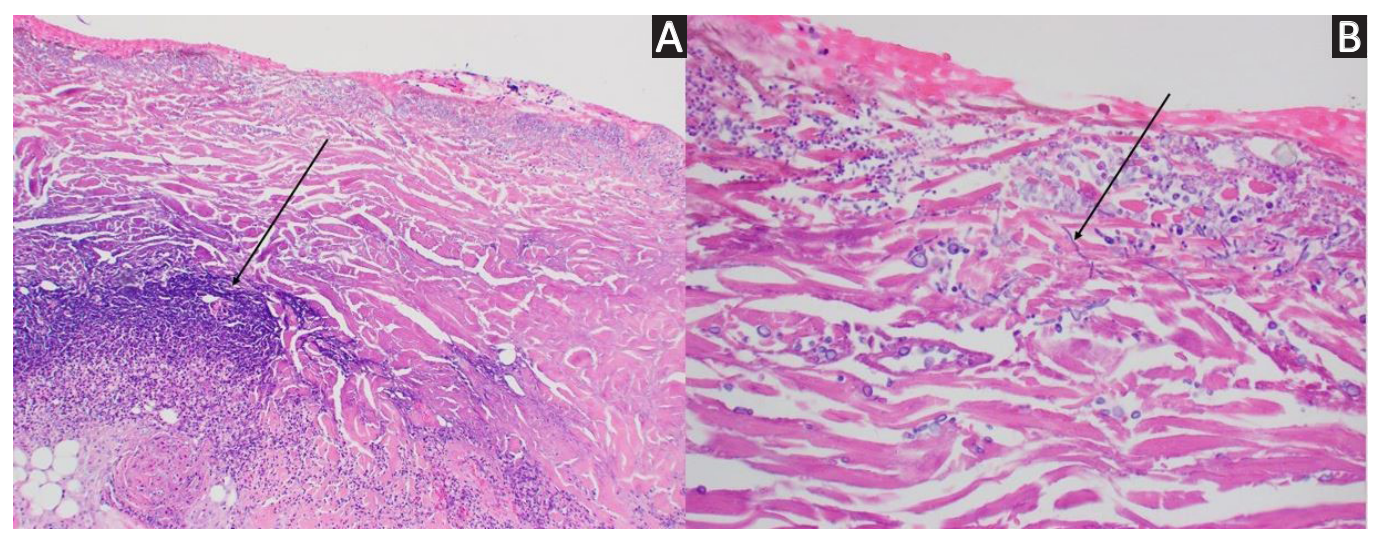

Fig. 2. (A) Histology from punch biopsy of perianal ulcers using haematoxylin and eosin stain showing suppurative necrosis at 10x magnification, and (B) with fungal elements at 40x magnification. 
Intravenous Augmentin 1.2g TDS and oral fluconazole $100 \mathrm{~g}$ OD were commenced. In view of the extensive skin necrosis, we performed definitive aggressive surgical debridement of the necrotic ulcers (Fig. 1B), with vacuum-assisted closure therapy applied post-excision. A rectal tube was placed for non-surgical faecal diversion. Subsequent intraoperative tissue culture grew Pseudomonas aeruginosa, Escherichia coli and Acinetobacter baumannii. The patient was continued on culture-directed antimicrobial treatment (IV Augmentin, oral fluconazole) for a total of 4 weeks. Her wound healed well with vacuum-assisted closure therapy after 2 months.

Although ecthyma gangrenosum usually occurs in patients who are critically ill and immunocompromised, it may develop even in the absence of bacteraemia and in immunocompetent person as illustrated in this case. Hence, ecthyma gangrenosum should be considered as a possible diagnosis even in a healthy patient especially when the ulcer is painless, rapidly progressing with characteristic central black eschar surrounded by an erythematous halo. ${ }^{1-3}$ Primary aspergillosis resulting in ulceration with secondary bacterial infection is another likely differential as morphology and appearance of the ulceration is very similar to ecthyma gangrenosum. In this case, the rapid progression of disease and involvement of the perineum points would favour the diagnosis of ecthyma gangrenosum. Often this condition should resolve with appropriate targeted antimicrobial treatment upon diagnosis by tissue culture and microscopic examination..$^{1-3}$ However, in view of the extensive skin involvement in this case, surgical debridement of the necrotic ulcers, with vacuum-assisted closure therapy applied post-excision were done with successful outcome.

\section{REFERENCES}

1. Somer T, Finegold SM. Vasculitides associated with infections, immunization, and antimicrobial drugs. Clin Infect Dis 1995;20:1010-36.

2. Zomorrodi A, Wald ER. Ecthyma gangrenosum: considerations in a previously healthy child. Pediatr Infect Dis J 2002;21:1161-4.

3. Sarkar S, Patra AK, Mondal M. Ecthyma gangrenosum in the periorbital region in a previously healthy immunocompetent woman without bacteremia. Indian Dermatol Online J 2016;7:36-9.

Yi-Quan Tan ${ }^{1}$ MRCS, Frederick H Koh $^{2}{ }^{F R C S E d}$, Choon-Sheong $\underline{\text { Seow }}^{3}$ FRCS (Glasg)

\footnotetext{
${ }^{1}$ Department of Urology, University Surgical Cluster, National University Hospital, Singapore

${ }^{2}$ Department of Colorectal Surgery, Division of Surgery, Sengkang

General Hospital, Singapore

${ }^{3}$ Division of Colorectal Surgery, Department of General Surgery,

Ng Teng Fong General Hospital, Singapore
}

Correspondence: Dr Frederick H Koh, Department of Colorectal Surgery, Sengkang General Hospital, 110 Sengkang East Way, Singapore 544886. Email: frederickkohhx@gmail.com 